Revue de l'Institut des langues et cultures

d'Europe, Amérique, Afrique, Asie et Australie

13 | 2010

Les voies incertaines de la démocratisation

\title{
Des démocraties en trompe l'œil ? Inégalités sociales et consolidations démocratiques en Amérique latine
}

\section{Charles Lancha}

\section{OpenEdition}

\section{Journals}

Édition électronique

URL : http://journals.openedition.org/ilcea/901

DOI : 10.4000/ilcea.901

ISSN : 2101-0609

\section{Éditeur}

UGA Éditions/Université Grenoble Alpes

Édition imprimée

ISBN : 978-2-84310-181-6

ISSN : 1639-6073

\section{Référence électronique}

Charles Lancha, « Des démocraties en trompe l'œil ? Inégalités sociales et consolidations démocratiques en Amérique latine », ILCEA [En ligne], 13 | 2010, mis en ligne le 30 novembre 2010, consulté le 20 avril 2019. URL : http://journals.openedition.org/ilcea/901 ; DOI : 10.4000/ilcea.901

Ce document a été généré automatiquement le 20 avril 2019

(C) ILCEA 


\title{
Des démocraties en trompe l'œil? Inégalités sociales et consolidations démocratiques en Amérique latine
}

\author{
Charles Lancha
}

1 La problématique de ma communication est, comme chacun le devine aisément, extrêmement vaste et complexe. Je ne saurais donc prétendre, en peu d'espace, en présenter une vision exhaustive. Mon propos est plus circonscrit. Il s'agit pour moi d'en dégager les principales orientations susceptibles de donner à comprendre comment la démocratie a évolué et l'état des lieux aujourd'hui.

2 En préambule à cette réflexion, il convient de rappeler que les dictatures des années 1960 et 1970 ont eu un double objectif: éliminer, exterminer les forces de gauche par une répression impitoyable mais aussi, on l'oublie souvent, imposer un nouveau modèle économique néolibéral, symbolisé au Chili par le rôle joué par Milton Friedman et les Chicago Boys et, en Argentine, par Martinez de Hoz. L'Amérique latine est devenue le laboratoire du néolibéralisme avant même son implantation en Grande-Bretagne par Margaret Thatcher en 1979 et aux États-Unis par Ronald Reagan à partir de 1981.

3 Les dictatures ont fini par être balayées par le mouvement populaire et on a assisté au retour de la démocratie mais le modèle économique néolibéral s'est perpétué et, comme on va être conduit à le montrer, il a dénaturé les régimes démocratiques au point qu'aujourd'hui on observe un double processus en Amérique latine: dans certains pays, conséquence des résultats économiques et sociaux catastrophiques, les peuples manifestent un net désenchantement vis-à-vis de la démocratie; dans d'autres cas, comme au Venezuela, en Bolivie ou en Équateur, les mouvements populaires ont porté au pouvoir des leaders comme Hugo Chavez, Evo Morales et Rafael Correa, qui rejettent le libéralisme et appellent à la création de démocraties conformes aux intérêts politiques, économiques et sociaux du plus grand nombre.

4 Sur le plan institutionnel, les démocraties ibéro-américaines se sont révélées des démocraties à part entière. Chaque pays comporte une constitution où les libertés fondamentales sont reconnues. Partout, des élections libres ont lieu périodiquement où 
s'affrontent un ou plusieurs partis au pouvoir et une opposition. L'alternance est une réalité ; l'indépendance du pouvoir judiciaire est affirmée, la liberté de la presse et des médias est proclamée. Bref, les régimes qui ont succédé aux militaires ont érigé des systèmes politiques qui, en théorie, n'ont rien à envier aux démocraties comme les ÉtatsUnis, la Grande-Bretagne ou la France. Si l'on a présent à l'esprit le style de gouvernement d'un Pinochet, d'un Videla ou d'un Díaz Ordaz, on ne peut qu'être sensible aux progrès considérables enregistrés dans le domaine des libertés publiques, conquises de haute lutte par les forces populaires.

5 On a donc bien à faire à des démocraties formelles mais à des démocraties minées par une série de tares. L'une d'elles a trait au manque de déontologie démocratique des élites dirigeantes. D'innombrables exemples pourraient être mis en avant pour illustrer leur fiasco. Retenons deux cas symboliques. Tout d'abord celui de l'Argentine. L'échec du retour à la démocratie éclate en 1989 de façon violente. L'expérience néolibérale du président Raul Alfonsin débouche sur une hyperinflation à l'origine d'une misère sociale généralisée qui donne lieu à des soulèvements du désespoir dans plusieurs villes et au pillage de supermarchés. L'État d'exception est décrété et la répression fait des dizaines de morts. Incapable de maîtriser la situation, Alfonsin met fin à son mandat avant l'heure.

Il passe le témoin à son adversaire politique, le péroniste Carlos Menem, qui conduit une campagne politique originale. Profitant du discrédit du radicalisme et de la confiance des travailleurs dans le péronisme, censé être le garant des intérêts de la classe ouvrière, il se fait élire sur le slogan "Suivez-moi », sans programme défini. À peine élu, il s'empresse d'appliquer, à la surprise générale et au pas de charge, un programme ultralibéral, dicté par le FMI, aux antipodes du péronisme orthodoxe. Les masses populaires font les frais de ce revirement alors que les capitalistes argentins et surtout les multinationales étrangères - au premier rang desquelles les espagnoles - font main basse sur le patrimoine argentin bradé à vil prix. Pratiquement toutes les conquêtes sociales du péronisme sont démantelées.

7 Menem, de plus, dilapide l'argent public et n'oppose aucun frein à la corruption. La dette extérieure de l'Argentine explose : entre 1993 et 1999, elle augmente de 28,5 \% à 41,5\% par rapport au PIB. Le chroniqueur du Monde, Paulo A. Paranagua a pu écrire à juste titre : «Le péroniste Carlos Menem, président de 1989 à 1999, a transformé l'Argentine en un État mafieux.»

8 Le radical Fernando de la Rúa qui succède à Menem témoigne d'une même duplicité politique. Élu le 24 octobre 1999 à la tête d'une alliance entre l'Union civique radicale (social-démocrate) et le FREPASO (Front pour un Pays solidaire, de centre gauche) sur la base d'un programme de lutte contre l'injustice sociale, il foule très vite aux pieds ses engagements électoraux. Comme ses prédécesseurs, il se plie aux diktats néolibéraux du FMI et de la Banque mondiale : réduction du déficit fiscal, baisse de 12 à $15 \%$ des salaires des 144000 fonctionnaires, licenciement de 10000 agents de l'État, dérégulation du travail.

9 De façon symbolique, en mars 2001, toute honte bue, il n'hésite pas à faire appel au ministère de l'Économie à Domingo Cavallo qui, pendant des années avait présidé, sous Menem, à la libéralisation à outrance de l'Argentine c'est-à-dire à la mise à sac du pays comme Fernando Solanas l'a magistralement dénoncé dans son film Mémoire d'un saccage.

Les plans d'austérité successifs de Cavallo se montrent incapables de surmonter la récession et ne font qu'accroître la pauvreté et la misère. En novembre 2001, l'économie 
argentine est au bord de l'asphyxie. La misère sociale est à son comble. Les 19 et 20 décembre 2001, lorsqu'il décrète le corralito, le gel des avoirs bancaires des épargnants, c'est l'émeute. Des émeutes de la faim éclatent en diverses villes du pays. En dépit de l'État de siège, les masses populaires se dressent contre le pouvoir pour exiger le départ du président. La répression se traduit par 27 morts, des centaines de blessés et plusieurs milliers d'arrestations. Pour Página 12 c'est «le mouvement civil de rébellion le plus important des 50 dernières années ». Le 21 décembre de la Rúa donne sa démission ${ }^{1}$ et laisse un pays dévasté économiquement et socialement. Pendant plusieurs semaines l'Argentine sombre dans le chaos et un président provisoire décrète un moratoire de la dette, c'est-à-dire la banqueroute du pays en cessation de paiement.

Le Pérou d'Alberto Fujimori offre un autre exemple caractéristique du peu de crédit que méritent les promesses électorales d'une grande partie des cercles dirigeants de l'hémisphère. Fils d'immigrés japonais, ce modeste ingénieur se présente aux élections présidentielles en avril 1990, soutenu seulement par une secte évangéliste et disposant de peu de moyens, il conquiert malgré tout la sympathie des déshérités, des laissés-pourcompte, qui se reconnaissent en cet homme simple, étranger au monde corrompu de la politique. Le discours de Fujimori recueille d'autant plus l'approbation d'une majorité d'électeurs qu'il paraît s'opposer à celui, redoutable, du candidat de la droite, l'illustre écrivain Mario Vargas Llosas. Ce dernier commet l'imprudence d'avouer que son programme néolibéral exigera dans un premier temps de durs sacrifices. Il déclare s'inspirer de Margaret Thatcher et de Reagan. Les masses populaires, déjà durement éprouvées par la crise, ne se sentent nullement portées à le soutenir. En revanche, elles se sentent beaucoup plus réceptives au message de l'immigré japonais centré sur la lutte contre la pauvreté. Le 12 juin 1990 Fujimori accède à la magistrature suprême.

12 À peine élu, il fait litière de ses engagements. À l'instigation du FMI, il met en pratique un plan d'ajustement draconien, le Fujichoc sans anesthésie qui se signale en particulier par une hausse de l'essence de $3000 \%$, la flambée des prix $-165 \%$ d'inflation - et l'effondrement du pouvoir d'achat. Comme conséquence, aux 7,5 millions de pauvres d'avril viennent s'en ajouter cinq autres. Pendant dix ans le Pérou connaît une démocratie dévoyée. Fujimori parvient à écraser la guérilla maoïste du Sentier Lumineux d'Abel Guzmán mais au prix d'une violation massive des droits de l'homme.

Le 28 mai 2000 Fujimori est réélu pour la troisième fois consécutive à l'issue d'un scrutin qualifié par les commentateurs de «farce électorale ». Sa carrière s'achève dans la honte : impliqué dans un scandale politique, il s'enfuit au Japon - sa patrie d'origine - où, le 19 novembre, il fait connaître sa démission. À l'égal de ses pairs argentins, il laisse un pays en ruine. Plus de la moitié des Péruviens sont alors réduits à l'indigence. Extradé du Chili, après 16 mois de procès, en avril 2009, Fujimori a été condamné à 25 ans de prison par un tribunal péruvien pour des crimes de lèse-humanité. Menem est également poursuivi par la justice argentine sous différents chefs d'inculpation.

Ces quelques exemples, parmi d'autres ${ }^{2}$, mettent en exergue les circonstances dans lesquelles les politiques néolibérales ont été imposées aux peuples argentin et péruvien : en contradiction avec toute déontologie démocratique. Aucun des présidents cités ne s'est fait élire sur un programme d'ajustement structurel conforme aux directives des organismes financiers internationaux. Tout au contraire.

15 Une autre tare touche aux ravages de la corruption au plus haut niveau de l'État. À titre d'exemples, on peut citer le cas de deux présidents qui, convaincus de s'être laissé corrompre, ont été destitués. Il s'agit de Fernando Collor de Mello, président du Brésil, et 
de Carlos Andrés Pérez, président du Venezuela. Le premier est déchu en 1992 et le second en 1993. D'autres noms pourraient être mentionnés comme celui de Fujimori - considéré par l'ONU comme l'un des dix hommes les plus corrompus au monde ${ }^{3}-$ ou celui de Arnaldo Aleman, président du Nicaragua de 1999 à 2001.

La corruption présente une gravité particulière dans la mesure où elle accentue la pauvreté des plus démunis ${ }^{4}$ et gangrène la plupart des sociétés latino-américaines. Le cas le plus dramatique est celui de la Colombie où la puissance financière considérable des narcotrafiquants leur a permis de dévoyer l'ensemble des institutions et de défier l'autorité de l'État. Le symbole le plus connu de l'emprise des parrains de la drogue c'est, évidemment, Pablo Escobar ${ }^{5}$. Aujourd'hui, en dépit du Plan Colombie financé aussi bien par Washington que par Bogota, l'ascendant économique et politique des cartels colombiens demeure extrêmement puissant. Mais c'est désormais le Mexique qui est dans l'œil du cyclone. L'armée mexicaine depuis des années a dû être engagée massivement dans la lutte contre les « narcos ». Des milliers de morts ont été enregistrés.

Les nouvelles démocraties sont par ailleurs affaiblies par leur incapacité à rompre totalement avec les dictatures militaires. Qu'il s'agisse de l'Argentine ou du Chili, de l'Uruguay, du Paraguay ou du Pérou, du Salvador ou du Guatemala, les anciens tortionnaires au pouvoir n'ont pu être jugés et condamnés dans leur ensemble. Pinochet est mort dans son lit. Videla, sous prétexte de son âge avancé purge sa condamnation à son domicile. Cette impunité est lourde de signification: elle exprime la puissance des forces de droite qui freinent toute avancée démocratique. Le cas du Chili est symptomatique $^{6}$.

18 Au-delà de toutes ces entraves à l'affermissement de la démocratie, l'essentiel réside dans la mise en œuvre pendant un quart de siècle dans toute l'Amérique latine, à l'exception de Cuba, d'un modèle économique néolibéral aux conséquences sociales désastreuses. Tous les gouvernements ibéro-américains aussi bien que la Banque mondiale ou l'OCDE dressent un même constat de faillite sur la situation sociale. En effet, l'Amérique latine connaît une ampleur généralisée de la pauvreté et de l'indigence, des inégalités abyssales au point que la Région est considérée comme la plus inégalitaire du monde. Rafael Dominguez Marin donne la mesure des inégalités en quelques chiffres: «En Amérique latine les $10 \%$ les plus riches reçoivent en moyenne $36 \%$ des revenus mais plus de $40 \%$ au Brésil, en Bolivie ou en Colombie ${ }^{7}$. » Le grand sociologue argentin Bernardo Kliksberg, conseiller de l'ONU, de la BID, de l'Unicef ou de l'Unesco, avance des chiffres plus sévères et plus fiables, extraits du rapport de la Banque mondiale de février 2006: «On estime que les $10 \%$ les plus riches possèdent $48 \%$ du revenu régional alors que les $10 \%$ les plus pauvres n'en perçoivent que $1,6 \%^{8}$.» Kliksberg souligne la gravité du phénomène qui affecte tous les domaines de la société :

L'inégalité latino-américaine ne s'exprime pas seulement dans la distribution des revenus. Elle s'applique à toutes les dimensions de la structure sociale, parmi elles l'accès à la propriété de la terre, la possibilité d'accès au crédit, les indicateurs vitaux les plus essentiels de la santé, de la mortalité infantile, de la mortalité maternelle, de l'espérance de vie, de l'accès à une éducation de qualité et à Internet ${ }^{9}$

Kliksberg a consacré une étude systématique et statistique de plus de 200 pages aux conséquences du libéralisme en Amérique latine ${ }^{10}$. C'est un réquisitoire tragique.

19 La faim est sans doute l'exemple le plus emblématique de l'acuité du déficit social propre à l'Amérique latine. On compte actuellement dans la Région, selon les chiffres tout récents du Programme alimentaire mondial 57 millions de sous-alimentés ${ }^{11}$. Ce fléau 
frappe tous les pays à des degrés divers et, à l'intérieur de chacun d'eux, il atteint des pourcentages très différents. Le seul pays épargné, selon les estimations de l'Unicef, est Cuba en raison des réponses gouvernementales appropriées apportées à ce problème ${ }^{12}$.

Les enfants comptent parmi les principales victimes de la faim. Pedro Medrano, directeur régional du PAM considère qu'un tiers d'entre eux souffrent de dénutrition (17 millions) ${ }^{13}$ . Il note que les gouvernements investissent 5 milliards de dollars dans des programmes alimentaires. 3 milliards supplémentaires permettraient de résoudre le problème mais «la volonté politique» fait défaut ${ }^{14}$. Les pays sont diversement affectés. Selon Mireya Mota, sous-directrice médicale au district fédéral de Mexico, on dénombrerait au Mexique 20 millions de personnes sujettes à une dénutrition chronique ${ }^{15}$.

21 Le cas du Guatemala est le plus sombre. L'Unicef s'indigne que $49,3 \%$ de la population infantile subissent une telle forme de dénutrition. Les enfants indigènes sont particulièrement concernés : 69,5\% d'entre eux ne mangent pas à leur faim ${ }^{16}$. La situation est également préoccupante en Colombie où le directeur régional de l'Unicef fait état de la mort, chaque année, de 20000 enfants dénutris. Elle l'est encore davantage en Bolivie : l'Unicef a diagnostiqué que $25 \%$ d'enfants de moins de deuxans y souffrent de dénutrition ${ }^{17}$. Le directeur régional juge que, en Bolivie et au Pérou, le nombre des décès est le double ou le triple de celui de la Colombie ${ }^{18}$.

$22 \mathrm{Au}$ vu de cette approche schématique de la faim en Amérique latine et du tableau de Bernardo Kliksberg, on comprend davantage que la thématique de la lutte contre la pauvreté et l'exclusion figure en tête des priorités gouvernementales. C'est ainsi que, au $17^{\mathrm{e}}$ sommet de la Communauté des nations ibéro-américaines, en novembre 2007, les chefs d'État se sont engagés, dans une déclaration solennelle, à promouvoir des politiques pour réduire la pauvreté et favoriser la cohésion sociale. Au-delà des intentions éthiques mises en avant, des impératifs politiques expliquent cet intérêt en faveur de la justice sociale.

On notera que, cinq ans plus tôt, les Nations unies avaient tiré le signal d'alarme. Dans un ouvrage publié sous leurs auspices, auquel avaient collaboré un grand nombre d'hommes politiques de premier plan, d'économistes, de sociologues, on pouvait lire dans le prologue :

L'existence du régime démocratique durant plus de deux décennies n'a pas été accompagnée d'avancées palpables en matière sociale. Tel est, en une phrase, l'apport central de notre rapport La democracia en America latina. Hacia una democracia de ciudadanas y ciudadanos... comment faire en sorte que l'égalité devant les urnes soit aussi une égalité des chances face à la vie ${ }^{19}$.

$24 \mathrm{Au}$ cours $\mathrm{du} 17^{\mathrm{e}}$ sommet qui se tenait à Santiago du Chili, la présidente Michelle Bachelet a évoqué les dangers pour la démocratie d'une telle situation :

Nous devons reconnaître que la dimension sociale n'a pas eu la même priorité que la démocratisation politique ou la modernisation économique au cours de ces dernières années. Cette réalité non seulement explique les énormes injustices mais pourrait finir par miner la légitimité de nos démocraties que nous avons eu tant de mal à construire ${ }^{20}$.

La Banque mondiale se montre plus précise sur le problème capital de l'Amérique latine d'aujourd'hui : «Transformer l'État en un agent qui dynamise l'égalité des chances et pratique une redistribution efficace est peut-être le défi le plus urgent qu'affronte l'Amérique latine. ${ }^{21}$ " Cette prise de position traduit un virage à $180^{\circ}$ de la Banque mondiale qui, dans les années 1980 et 1990, proclamait la nécessité absolue du 
dépérissement de l'État et avait pour dogme que la croissance économique par elle-même aurait la vertu de diminuer la pauvreté. C'était la théorie du "derrame », de l'essaimage de la richesse. Grossière erreur. C'est à partir de 2005 que la Banque mondiale a fait son autocritique $^{22}$.

De fait, d'énormes attentes sociales existent et se manifestent en Amérique latine. Si elles venaient à être une fois de plus déçues, certains n'excluent pas que les démocraties risqueraient d'être de ce fait menacées. Georges Couffignal prend acte que, d'après les chiffres de la CEPAL, le retour de la croissance dans la Région a été accompagné d'un léger recul, en pourcentage, de la pauvreté. Mais il observe que, dans le même temps, «le nombre de pauvres est passé de 136 millions (dont 62 extrêmement pauvres) à 205 millions (dont 79 millions extrêmement pauvres), soit une augmentation de 69 millions de pauvres (dont 17 millions extrêmement pauvres) en un quart de siècle ${ }^{23}$ ». Il conclut : « On comprend mieux dès lors la désaffection des populations vis-à-vis de la démocratie que soulignent régulièrement les enquêtes annuelles du Latinobarómetro, une organisation non gouvernementale établie à Santiago. » Raimón Obiols opine dans le même sen $\mathrm{s}^{24}$. Ce point de vue est également partagé, dans la même revue, par Renée Fregosi - déléguée nationale chargée des intégrations régionales en Amérique latine du parti socialiste -, p. 122 .

Le paupérisme n'est pas le seul défi gigantesque auquel l'Amérique ibérique est confrontée. Il en est un autre tout aussi redoutable : la criminalité galopante qui revêt deux formes : la délinquance et le crime organisé. Bernardo Kliksberg a établi une nette ligne de démarcation entre ces deux formes de violence :

D'un côté il y a le crime organisé, sous ses différentes formes, les bandes de drogués, les mafias, les groupes de kidnapping, les organisations de trafic d'êtres humains, de vols d'automobiles, de blanchiment d'argent sale. Il est clair que la société doit s'en défendre et faire peser sur elle tout le poids de la loi... D'un autre côté, on assiste à la croissance très forte d'une criminalité chez les jeunes qui débute par des actes de petite délinquance de la part d'enfants et d'adolescents en situation de grande vulnérabilité. Si on la sanctionne de la même manière que le crime organisé... toutes les conditions qui les ont conduits à la délinquance vont empirer. On ne fera que fournir de "la chair à canon » au crime organiséé 25 .

$\mathrm{Au}$ cours du dernier quart de siècle, on ne peut qu'être frappé par l'extension de la violence multiforme dans le sous-continent. Selon un correspondant permanent de El País , Francesc Relea : «Aucune région au monde n'est confrontée à des niveaux de violence liée à la délinquance et à la criminalité ordinaire comparables à ceux de l'Amérique latine ${ }^{26}$.» Les causes de cette violence sont multiples. Elles tiennent à la misère, au fait que le chômage frappe massivement la jeunesse et la rend plus vulnérable à la délinquance, au trafic de drogue et aux séquelles de la guerre civile en Amérique centrale. Le narcotrafic est générateur de criminalité. La Colombie en est la sinistre illustration. Au cours des dernières décennies, les parrains de la drogue se sont enrichis fabuleusement et acquis un tel pouvoir qu'ils ont corrompu toutes les institutions, se sont transformés en un véritable État dans l'État. Lorsque, par exemple, le gouvernement colombien, sous la pression des États-Unis, a tenté de les éliminer, c'est une véritable guerre qu'il a dû livrer. En 1990, les affrontements avec les mafieux de la cocaïne se soldèrent par 13000 morts ${ }^{27}$. En 1993, en six mois, on enregistra 3600 victimes. En 1995, les attentats des narcotrafiquants et la répression entraînèrent 20000 morts et 700 enlèvements. Des dizaines de journalistes, des centaines de juges, des milliers de policiers ou de soldats, des hommes politiques de premier plan ont été assassinés par les hommes de main - les 
« sicarios »- des cartels de Medellin ou de Cali. Alliés aux grands propriétaires terriens, les narcotrafiquants ont constitué des escadrons de la mort qui ont assassiné systématiquement des milliers de militants communistes de l'Union patriotique. Comme chacun sait, la violence sévit toujours en Colombie. Le cas d'Ingrid Bétancourt est dans toutes les mémoires. En 2008, 48 personnes ont trouvé la mort en moyenne chaque jour de façon violente en Colombie ${ }^{28}$. Jusqu'au mois d'avril 2009, 1009 poursuites ont été engagées contre des membres de l'Armée, de la Marine, de la police et du Service de renseignement pour l'assassinat de 1666 civils sans défense. En mai, le Procureur Mario Iguaran a informé l'opinion publique que 426 militaires ayant commis des exécutions extrajudiciaires étaient incarcérés ${ }^{29}$.

Dans ce martyrologe, les syndicalistes occupent une place particulière. Dans le dernier rapport de la Confédération syndicale internationale sur la situation des droits syndicaux, il apparaît que la Colombie demeure le pays le plus dangereux pour les syndicalistes. Sur 76 syndicalistes assassinés dans le monde en 2008, 49 l'ont été en Colombie - 10 de plus qu'en $2007^{30}$. Dans une impunité presque totale ${ }^{31}$.

30 La violence affecte tous les pays d'Amérique latine, à des degrés divers. Le Mexique est particulièrement touché. Depuis plusieurs années, la criminalité s'est accrue de façon vertigineuse. À la fin des années 1990 on relevait chaque année 1900 enlèvements en vue d'extorquer une rançon aux familles des victimes. Depuis 2007 le président Felipe Calderon a engagé l'armée, 40000 hommes, dans la lutte contre les narcotrafiquants. En pure perte. Le trafic de drogue ne faiblit pas. En revanche, les violations des droits de l'homme ont pris une ampleur considérable ${ }^{32}$.

31 Les femmes paient un lourd tribut à la criminalité. La presse internationale s'est fait largement l'écho des centaines de jeunes femmes assassinées depuis près de quinze ans à Ciudad Juarez, à la frontière avec les États-Unis ${ }^{33}$. En toute impunité ou presque. On parle désormais de féminicide. On retrouve ce même phénomène en Amérique centrale. $\mathrm{Au}$ cours des dernières années, plus de 2000 femmes ont été assassinées au Guatemala ${ }^{34}$. Là encore, pratiquement en toute impunité.

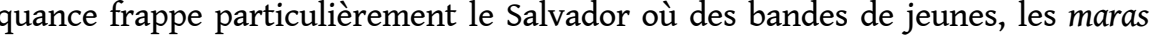
sévissent et font régner un climat d'insécurité permanent. Dans ce petit pays, deux millions d'armes circulent sans contrôle. Cinq mille jeunes y sont sous les verrous ${ }^{35}$. Les cinq présidents de l'isthme centre-américain - Guatemala, Costa Rica, Nicaragua, Salvador et Panama - ont consacré un sommet à la répression du crime organisé le $1^{\mathrm{er}}$ avril 2005 à Tegucigalpa, en présence de représentants des États-Unis et du Mexique.

Dans l'immense Brésil, la situation de la criminalité est tout aussi préoccupante. Un chiffre parle de lui-même: chaque année, on compte au Brésil 30000 morts violentes. Cela depuis 20 ans: 600000 victimes en deux décennies ${ }^{36}$. Rio et Sao Paulo qui rassemblent $18 \%$ de la population totale du pays concentrent $40 \%$ de la violence. À Sao Paulo, on comptait en 20021000 homicides par mois ${ }^{37}$. À titre de comparaison, rappelons que l'« on compte 1000 homicides par an en France et à peu près autant de tentatives ${ }^{38}$ ».

34 La police, incapable de faire front à la criminalité galopante, recourt à tous les moyens pour y faire face. En 1999 les policiers de Sao Paulo ont ainsi abattu, en invoquant la légitime défense, 650 personnes, invariablement présentées comme de dangereux malfaiteurs. Une hécatombe qui masque, en réalité, les exécutions sommaires de jeunes délinquants ${ }^{39}$. Le 8 janvier 2004 Le Monde titrait un grand article: "Au Brésil, les 
homicides déciment la population jeune, noire et pauvre». Bartolomé Bennassar et Richard Marin établissent le même diagnostic: "La mort ordinaire a les traits d'un homme jeune, entre dix-huit et vingt-quatre ans, noir ou mulâtre, pauvre et plutôt banlieusard ${ }^{40}$.» Les années ont passé mais la situation ne s'est pas améliorée, comme l'illustre ce témoignage de Jean-Pierre Langellier en 2008 : « Les chiffres de la violence au Brésil donnent le vertige. Il y a 50000 homicides par an. L'État de Rio bat des records. En sept mois, de janvier à septembre 2007, la police a tué 1300 personnes, soit $60 \%$ de plus que l'année précédente. Chaque jour, trois jeunes (15-24 ans) sont assassinés ${ }^{41}$. "

En août 2000, le gouvernement brésilien a reconnu à l'ONU qu'il n'était pas parvenu à éradiquer l'usage de la torture par les forces de l'ordre ${ }^{42}$. La police brésilienne est considérée comme une des plus violentes au monde ${ }^{43}$.

$\mathrm{Au}$ vu de la radiographie que nous venons d'esquisser de la démocratie en Amérique latine, il est compréhensible qu'un certain nombre de peuples aient opté dans les urnes pour un changement politique dans l'espoir que s'affirment des gouvernements davantage à l'écoute de leurs revendications sociales.

$\mathrm{Au}$ cours de la première décennie du XXI $\mathrm{e}^{\mathrm{e}}$ siècle, la gauche a ainsi accédé au pouvoir dans la plupart des pays du sous-continent, puis au Nicaragua et, tout dernièrement, au Salvador. Au Brésil, Luiz Inacio Lula da Silva, fondateur du Parti des travailleurs, a été élu président de la République le 27 octobre 2002 avec $61 \%$ des suffrages exprimés puis réélu le 29 octobre 2006 pour un second mandat. En Argentine, le péroniste Nestor Kirchner a été désigné à la magistrature suprême le 25 mai 2003 ; sa femme, Cristina Fernandez lui a succédé, élue le 28 octobre 2007 ; Hugo Chavez est devenu président du Venezuela le 2 février 1999 et a été réélu en 2000 puis en 2006; au Chili, le socialiste Ricardo Lagos a occupé le Palais de la Moneda du 11 mars 2000 au 11 mars 2006 et Michelle Bachelet, également socialiste, lui a succédé le 15 janvier 2006 pour un mandat de quatre ans; en Uruguay, à la tête d'une coalition de gauche, Tabaré Vazquez a triomphé à la présidentielle le 31 octobre 2004 et son mandat s'achèvera le $1^{\mathrm{er}}$ mars 2010 ; en Bolivie, Evo Morales a été élu chef de l'État le 18 décembre 2005, élection historique où, pour la première fois un Indien a accédé à la présidence de la nation; en Équateur, Rafael Correa a été élu président le 26 novembre 2006 et, en novembre de la même année ce fut le cas du Nicaraguayen Daniel Ortega, leader du sandinisme; la présidence du Paraguay est échue à Fernando Lugo, "l'évêque des pauvres", le 20 avril 2008; au Salvador, le représentant du FMLN - Front Farabundo Marti de Libération Nationale - Mauricio Funes a remporté les élections présidentielles le 15 mars 2009.

Il est bien connu que tous ces gouvernements de gauche ne manifestent pas les mêmes options politiques. En Amérique du Sud, la ligne de partage s'établit entre, d'une part le Brésil, le Chili et l'Argentine, considérés comme modérés et, d'autre part le Venezuela, la Bolivie et l'Équateur, antilibéraux, anti-impérialistes et orientés vers le socialisme. Hugo Chavez est le chef de file indiscutable de la contestation révolutionnaire en Amérique latine. Le Venezuela et la Bolivie ont constitué avec Cuba une Alliance, l'ALBA - Alternative bolivarienne des Amériques - à laquelle divers pays se sont joints: le Nicaragua et le Honduras, la Dominique, Saint-Vincent et Grenadines. L'Équateur et le Paraguay y figurent à titre d'observateurs.

39 Faute de temps, il n'est pas possible d'exposer les positions adoptées par les différents gouvernements progressistes. Nous nous en tiendrons ici à celles du Brésil et de la République bolivarienne du Venezuela qui témoignent des choix idéologiques de chacun des deux groupes en présence. 
40 Le président brésilien Lula est l'illustration d'un cas classique en politique : le cas d'un candidat à la présidentielle qui se fait élire sur un programme de gauche et, une fois élu, pratique une politique centriste. Ce revirement est d'autant plus frappant que Lula est une personnalité d'exception. D'origine très modeste, il eut faim dans son enfance comme c'est le sort aujourd'hui de 50 millions de Brésiliens, il est devenu ouvrier métallurgiste, a assumé de hautes responsabilités syndicales, s'est dressé contre la dictature militaire et a contribué de manière décisive à la création du parti de gauche, le Parti des travailleurs.

41 Dans ces conditions, il n'est pas étonnant que, en 2001, lorsque - pour la quatrième fois il se porte candidat à l'élection présidentielle et que les sondages le déclarent favori, l'oligarchie nationale et internationale soit prise de panique.

Quelques mois avant l'élection, le Brésil se trouve en situation de quasi-faillite, comme l'Argentine à l'époque. Le FMI assure la sortie de crise par une injection de capitaux mais, en contrepartie, il obtient l'engagement formel des candidats - et notamment de Lula d'entreprendre des coupes sombres dans les dépenses publiques. Lula, après son succès électoral, a dû choisir des ministres pro-FMI et sa politique monétariste s'est inscrite dans la continuité par rapport à celle de son prédécesseur, Fernando Henrique Cardoso. La priorité absolue, dictée par le FMI, a été le paiement scrupuleux des intérêts de la dette. Une dette considérable : fin 2004, elle se montait à 360,6 milliards de dollars, soit $51,9 \%$ du PIB ${ }^{44}$. Les intérêts de la dette payés en 2004 se sont élevés à plus de 48 milliards de dollars ${ }^{45}$.

Un tel carcan financier et la rigueur consécutive ont interdit la mise en œuvre effective de la politique sociale annoncée au cours de la campagne électorale : amélioration de l'emploi et des salaires les plus bas, mise en place de réformes structurelles en faveur d'une meilleure égalité sociale et aide immédiate aux plus démunis par le Plan Faim Zéro. Toutes ces réformes sont restées à l'état d'ébauches. Retenons trois exemples significatifs.

En 1998, Lula proclamait que le salaire minimum devrait être de 1100 reaís (330 euros). En 2004, il octroie une augmentation de 20 reaís: le salaire minimum passe de 240 à 260 reaís (78 euros) puis, en janvier 2005, à 300 reaís ( 87 euros ${ }^{46}$. Cette même année, il n'ose participer à aucune manifestation du $1^{\mathrm{er}}$ mai par peur d'être hué $e^{47}$. En 2006, le salaire minimum a été porté à 350 reaís (150 euros). Il a été relevé de $12 \%$ en février 2009, à 615 reaís par mois soit 205 euros environ ${ }^{48}$.

Autre exemple : la réforme agraire. Peu avant le scrutin, Lula avait déclaré : «Si je ne fais qu'une chose comme président, ce sera la réforme agraire. » Les mesures adoptées en la matière n'ont guère donné satisfaction aux intéressés, les paysans sans terre, environ 4 millions de familles ${ }^{49}$. Du 12 au 17 mai 2005, 12000 paysans ont entrepris une Marche nationale pour la réforme agraire. Ils ont parcouru $233 \mathrm{~km}$, de Goiânia - ville du CentreOuest - à Brasilia. En 2003 et 2004, 117000 familles ont reçu des terres du gouvernement. Cependant, pour $79 \%$ d'entre elles, c'est le résultat des projets de réforme agraire du précédent président, Cardoso. Le gouvernement actuel n'a en réalité accordé une terre qu'à 25000 familles. Officiellement, 80000 familles ont reçu une terre en deux ans et demi, alors que Lula s'était engagé à en distribuer à 110000 familles par an ${ }^{50}$.

Où en est-on aujourd'hui ? Le ministre brésilien de la réforme agraire, Guilherme Cassel, assure que le programme initial a été couronné de succès et que 520000 familles en ont bénéficié au cours des six premières années de la présidence de Lula. Il ajoute qu'entre 70000 et 100000 familles par an se verront attribuer une parcelle de terre ${ }^{51}$. Le MST récuse ces statistiques. Pour lui, ce sont un peu moins de 100000 familles qui sont 
devenues propriétaires de leur sol. Par ailleurs, il subsisterait 4 millions de paysans sans terre et 230000 familles attendraient toujours la réforme agraire, installées dans des campements précaires.

Le gouvernement n'a pris aucune mesure contre le latifundium qui domine les campagnes : $1 \%$ des fazendeiros détient $45 \%$ des terres ${ }^{52}$. Comme l'agrobusiness est très performant au Brésil et que, en 2004, l'excédent de la balance commerciale pour le seul secteur de l'agriculture s'est chiffré à 34 milliards de dollars, Lula n'a pas jugé bon de porter atteinte à ses intérêts. Une journaliste du Monde propose le commentaire suivant : "On ne tue pas la poule aux œufs d'or ${ }^{53}$.»

Des changements structuraux décisifs se sont effectivement produits dans l'agriculture brésilienne qui n'ont pas échappé au leader charismatique du Mouvement des Sans-terre, João Pedro Stedile. Il met en exergue la montée en puissance des multinationales, à la faveur du néolibéralisme, et la nécessité pour le prolétariat rural d'innover ses formes de lutte:

La réforme agraire traditionnelle consistait à détruire les latifundia et à se partager la terre. Ce type de réforme à lui seul ne suffit plus. Le néolibéralisme l'a dynamité. Les bourgeoisies nationales ne dépendent plus des marchés internes. Elles ont négocié avec les multinationales et travaillent maintenant pour le marché extérieur. Elles imposent des monocultures, détruisent l'environnement, monopolisent la vente des semences, disposent des patentes pour les transgéniques. Alors, à quoi bon détruire des latifundia et se partager les terres si nous ne possédons pas de semences à y planter? Le problème est devenu plus complexe et l'axe de notre lutte doit changer ${ }^{54}$.

Troisième exemple : le Plan Faim Zéro. Lula avait fait de la lutte contre la faim - qui affecte de 45 à 50 millions de Brésiliens - sa priorité $n^{\circ} 1$ lors de sa campagne électorale. À plusieurs reprises il déclara: «Si au terme de mon mandat chaque Brésilien peut faire trois repas par jour, j'aurai accompli la mission de ma vie. » Dans un premier temps, le gouvernement avait envisagé de verser dans le programme Faim Zéro 100 milliards de reaís (30 milliards d'euros) mais la contrainte budgétaire exigée par le FMI, en échange d'un prêt de 30,4\% milliards de dollars, a limité à environ 500 millions d'euros les fonds destinés en 2003 au plan. Les familles concernées par Faim Zéro reçoivent 50 reaís (environ 13 euros) par mois et, en contrepartie, s'engagent à vacciner leurs enfants et à les scolariser ${ }^{55}$.

51 L'organisation du plan a conduit à la mise en place d'une gigantesque machinerie administrative qui nuit à son efficacité. De nombreuses familles concernées tardent à toucher les subsides. Ce programme, finalement, n'a pas réussi à atteindre totalement ses objectifs. Son bilan est mitigé selon «Chico» Whitaker, l'un des fondateurs du Forum social mondial : «Des millions de personnes bénéficient déjà de ce programme mais c'est insuffisant. Le problème c'est qu'on s'attaque aux effets, pas aux causes. »

Selon François d'Arcy, l'échec du plan Faim Zéro en raison de son impréparation a conduit à l'adoption du programme de substitution Bolsa Familia - allocation-famille présenté comme «le programme phare de la politique sociale ${ }^{56}$ » qui fusionne le programme «carte-alimentation» et trois autres programmes du temps de Fernando Henrique Cardoso.

À propos du premier mandat de Lula, au Brésil, Marcos Ceballos et Bruno Lautier tirent cette conclusion: "La Bolsa Familiaa peu d'effets sur la proportion de pauvres en raison d'un seuil d'attribution très bas et du faible niveau de ces allocations. » Dans le même 
ouvrage, Amérique latine: les surprises de la démocratie, François d'Arcy établit un «bilan contrasté » de la première présidence de Lula. À propos de la Bolsa Familia, il relève la médiocrité des subsides :

les prestations versées sont restées modestes par rapport aux autres prestations sociales organisées par la Constitution de 1988 et qui concernent principalement les personnes âgées ou handicapées: alors que celles-ci sont alignées sur le salaire minimum qui a été porté à 350 reaís (130 euros) - 145 dollars en Argentine - en 2006, les prestations au titre de Bourse Famille varient entre 15 et 95 reaís par famille avec enfant(s) et n'ont pas augmenté depuis 2003. (p. 41)

À propos des «politiques universelles d'enseignement et de santé tout aussi importantes pour combattre la pauvreté », François d'Arcy se montre tout aussi critique : "les performances du gouvernement Lula en ces domaines sont décevantes ».

En Argentine, comme au Brésil, les subsides versés sont faibles. Selon Ceballos et Lautier : «Depuis mars 2006, les familles perçoivent un revenu mensuel de base de 150 pesos (48 dollars) plus 25 pesos ( 8 dollars) par enfant de moins de 19 ans ou ayant un handicap, avec un plafond de 300 pesos mensuels (97 dollars).» (p. 71)

Ces prestations de survie, aussi faibles soient-elles, ont bénéficié électoralement aussi bien à Lula qu'à Cristina Kirchner. Pourtant, la fameuse promesse de campagne de Lula d'abolir la faim au Brésil n'a pas été vraiment tenue.

De façon symbolique, au Brésil, les enfants pauvres ne demandent pas de cadeaux de Noël à leurs parents. Ils savent qu'ils ne peuvent leur en acheter. La coutume veut, à Recife, qu'ils envoient leurs lettres au Papa Noël de la Poste. La Poste a reçu en décembre 2007 10000 lettres d'enfants, 6000 y demandaient de la nourriture pour Noël ${ }^{57}$. Jean-Luc Mélenchon se montre trop optimiste sur les résultats de la politique sociale de Lula ${ }^{58}$. De même, on éprouve quelque difficulté à partager l'opinion suivante d'Alain Rouquier : "Ainsi, le Brésil de Lula parvient à concilier, dans un cadre démocratique, l'orthodoxie financière et la lutte contre la pauvreté. Il s'efforce de rendre compatibles croissance économique et justice sociale ${ }^{59}$.» Alain Rouquier note que les choix de l'ancien syndicaliste ne font pas l'unanimité, même parmi les siens : «Outre les déçus du Parti des travailleurs, une partie de l'opinion comprend mal les orientations du gouvernement Lula » (p. 315). Comment pourrait-il en être autrement? Il a beau jeu d'expliquer :

Que les pionniers de l'alter-mondialisme, créateurs du Forum social mondial, soient devenus les champions de la mondialisation surprend et désoriente. Que les hommes politiques qui ont, pendant dix ans, fustigé le «néolibéralisme» pratiquent l'orthodoxie financière la plus rigoureuse et réclament la libéralisation du commerce international au nom des avantages comparatifs n'est pas facile à justifier... Mais l'incompréhension est plus générale encore face au maintien et à l'aggravation de la rigueur financière et budgétaire. (p. 315-316)

Pour conclure sur Lula, on constate qu'il applique une politique de rigueur monétaire qui lui vaut les félicitations du FMI et des marchés financiers et la réprobation d'une partie des forces de gauche dont il est issu. Peu avant sa mort, le célèbre économiste Celso Furtado émettait le jugement suivant :

Forcer un pays qui ne satisfait pas encore les besoins minimums d'une grande partie de la population à paralyser des secteurs modernes de son économie, à ne pas investir suffisamment dans la santé et l'éducation pour respecter des ajustements de la balance des paiements imposés par des bénéficiaires de taux d'intérêt élevés est quelque chose qui échappe à toute rationalité.

Parallèlement à Lula, un autre leader de la gauche latino-américaine s'est hissé au premier plan de la scène régionale, Hugo Chavez, élu démocratiquement en 1998 et 
confirmé à plusieurs reprises par les urnes à la tête de la République bolivarienne. Lula et Chavez sont alliés et militent l'un et l'autre en faveur de l'unité sud-américaine mais leurs orientations idéologiques divergent.

Chavez s'est heurté au Venezuela à des problèmes sociaux comparables à ceux du Brésil : une société profondément inégalitaire où $70 \%$ de la population vit dans la pauvreté et où 800000 familles sont vouées à l'indigence.

Chavez a posé comme diagnostic que cet état de fait résultait de la convergence de trois facteurs : la concentration des richesses et la disparité des revenus, le néolibéralisme et l'impérialisme américain au service des multinationales.

À partir de ce constat, il a élaboré et commencé à mettre en pratique un programme aux lignes de force nettement marquées. En réponse à la misère du plus grand nombre, il a ouvert des écoles bolivariennes. Dans ces 3750 établissements, plus d'un million d'enfants pauvres reçoivent gratuitement, outre l'éducation, deux repas et deux goûters chaque jour, en plus de l'uniforme, des manuels et des cahiers. En 2004, le budget de l'éducation représentait $20 \%$ du budget national.

Un autre programme : la mission Barrios Adentro - Au cœur des quartiers - est sans doute le programme social le plus marquant. Grâce à un accord avec le gouvernement cubain, cette mission a installé 20000 médecins cubains dans les quartiers populaires qui dispensent des soins gratuitement. Quelque 1500 médecins vénézuéliens se sont intégrés à la mission.

En réplique à la concentration des richesses, Hugo Chavez s'est prononcé pour la réforme agraire, une réforme qui se traduit par l'expropriation des grands domaines improductifs. De même, il manifeste l'intention d'exproprier les entreprises inactives et de les confier aux travailleurs.

64 Toutes ces initiatives s'inscrivent dans la lutte contre la pauvreté, problème fondamental au Venezuela. Ont-elles porté leurs fruits? La pauvreté a-t-elle reculé? Comme on pourrait s'y attendre, compte tenu de l'entreprise de dénigrement systématique des médias occidentaux à l'égard du régime bolivarien, la grande presse n'a eu de cesse de prétendre qu'Hugo Chavez avait échoué et que la pauvreté s'était aggravée. Évidemment, le gouvernement vénézuélien soutient le contraire. Pour trancher, il y a lieu de se référer à une source objective qui fait autorité, en l'occurrence la CEPAL - Commission économique pour l'Amérique latine -, organisme relevant de l'ONU.

Selon la CEPAL, le taux de pauvreté au Venezuela a sensiblement baissé de 2002 à 2006 : il est passé de $48,6 \%$ de la population à $30,2 \%$, soit une diminution de $18,4 \%$. Même chose pour l'indigence qui a chuté de $22,2 \%$ à $9,9 \%$, soit une différence de $12,3 \%{ }^{60}$. En 2006 , $8,3 \%$ de Vénézuéliens disposaient d'un revenu inférieur à un dollar et $27,6 \%$ d'un revenu inférieur à deux dollars ${ }^{61}$.

En 2009, la secrétaire générale de la CEPAL, Ana Barcena, a donné sur CNN les informations suivantes sur la situation sociale au Venezuela : «Je peux confirmer que le taux de chômage a diminué de 11 à $7,4 \%$, que le taux de pauvreté extrême est passé effectivement de 25 à $8,5 \%$ en $2007 \ldots$ et que la pauvreté a baissé de 51 à $28 \%{ }^{62}$. » Elle donnait ainsi son aval aux chiffres proposés peu auparavant par Hugo Chavez. L'évolution de l'indice de développement humain élaboré chaque année par l'ONU pour les différents pays du monde corrobore les progrès enregistrés par le Venezuela ${ }^{63}$. 

s'est prononcé pour le socialisme, un socialisme qui, dans son esprit, reste à inventer : « Je suis convaincu que le chemin à suivre c'est celui du socialisme, non n'importe quel socialisme, c'est un défi, nous sommes obligés d'inventer le socialisme. » Dans l'immédiat, même si à Mar del Plata en Argentine, devant 40000 personnes, il a condamné le capitalisme, il apparaît que Chavez n'exclut nullement l'entreprise privée, mais favorise la formation d'entreprises mixtes associant l'État et des coopératives de travailleurs.

Son grand projet c'est, dans l'esprit de Bolivar - le grand inspirateur du régime - de contribuer à l'intégration de l'Amérique latine, une intégration fondée sur la solidarité et non sur « les vices du capitalisme ». Selon ses vues, seule une Amérique latine unie pourra tenir tête aux États-Unis, la puissance hégémonique : «L'unique voie de salut pour nos peuples est l'intégration politique, sociale, économique. »

L'unionisme bolivarien l'a conduit à nouer des liens étroits, solidaires avec Cuba, à créer avec Fidel Castro l'ALBA - Alternative bolivarienne des Amériques - en opposition à la Zone de libre échange des Amériques - ZLEA ou ALCA, sigle espagnol -, à s'agréger au MERCOSUR - Marché commun de l'Amérique du Sud -, à développer considérablement les liens commerciaux avec le Brésil, l'Argentine, à fonder l'organisme PetroCaraïbe auquel se sont associés 16 États insulaires qui reçoivent du Venezuela des fournitures de pétrole à des prix préférentiels.

70 En somme, Hugo Chavez se fait l'ardent avocat auprès des Latino-américains d'une alternative bolivarienne antilibérale opposée à l'Oncle Sam. Pour Washington, il est devenu le principal adversaire au sud du Rio Grande. Un adversaire d'autant plus redoutable que, selon l'écrivain argentin Tomas Eloy Martinez, peu suspect de sympathie à son égard, « Hugo Chavez est devenu la figure dominante en Amérique latine » et qu'il dispose d'un puissant moyen d'action, le pétrole.

71 À l'heure de conclure, on ne peut que se réjouir que la démocratie ait cours depuis un quart de siècle dans l'ensemble de l'Amérique latine, mais ce brevet de longévité invite-til pour autant à l'optimisme sur la nature profonde des démocraties latino-américaines du dernier quart de siècle? On peut en douter. Certes, d'un point de vue institutionnel, les régimes démocratiques ibéro-américains s'inscrivent dans la modernité et c'est une avancée exceptionnelle par rapport au passé. Cela étant, les ombres ne manquent pas au tableau. Une pauvreté, une misère de grande ampleur qui entraînent des crises et l'instabilité politique. Un nombre significatif de chefs d'État n'ont pu parvenir au terme de leur mandat, contraints à la démission par la rue, par des explosions sociales. Autre ombre majeure : la violation massive des droits de l'homme. Nous en avons relevé un certain nombre d'exemples. Nous aurions pu en citer bien d'autres.

Olivier Dabène s'interroge sur la qualité de la démocratie latino-américaine. À l'évidence, elle laisse beaucoup à désirer. On est en droit de s'interroger : s'agit-il bien d'authentiques démocraties ou plutôt de démocraties en trompe-l'œil, autrement dit de démocraties apparentes mais trompeuses? La réponse ne va pas de soi. On est fondé à considérer que la pauvreté et l'indigence qui frappent massivement tous les pays de l'hémisphère entraînent une exclusion de fait de la citoyenneté d'une proportion considérable de la population, parfois majoritaire, comme au Guatemala ${ }^{64}$, si l'on pose en principe que la démocratie ne saurait se réduire à la seule liberté de suffrage. En dehors du droit de vote, de quels autres droits bénéficient les masses populaires victimes d'un déficit social gigantesque, comme les élites elles-mêmes l'admettent aujourd'hui ${ }^{65}$ ? Elles jouissent d'un 
droit qu'elles ont conquis par l'action : celui de s'insurger contre un ordre social injuste, de se doter d'organisations syndicales, politiques propres qui portent leurs revendications. Les démocraties latino-américaines dressent d'énormes obstacles à l'expression de la vox populi mais ces obstacles peuvent être franchis. C'est la leçon qui se dégage de la victoire de Hugo Chavez, de Lula, de Evo Morales, de Rafael Correa, de Tabaré Vazquez, de Daniel Ortega, de Fernando Lugo ou de Mauricio Funes. Pendant 25 ans, les classes dirigeantes se sont employées à cadenasser les régimes démocratiques en leur faveur mais le modèle libéral imposé par elles vole en éclats de plus en plus, contesté de toutes parts dans le cadre de la crise du capitalisme à l'échelle mondiale. Et la voie s'est ouverte au cours de la dernière décennie à l'émergence en Amérique latine de nouvelles démocraties où le pouvoir cesse d'être accaparé par une minorité de privilégiés inféodée au consensus de Washington et où les légitimes revendications des masses populaires obtiennent des réponses appropriées.

\section{NOTES}

1. De la Rúa n'est pas le seul président latino-américain à avoir dû mettre un terme à ses fonctions sous la pression de la rue. On en compte dans une époque récente trois en Bolivie, deux en Équateur et un à Haïti. Maurice Lemoine en dénombre quatorze depuis le début des années 1980. (Le Monde Diplomatique, mai 2009, p. 16.)

2. Le cas d'Alan García, élu en 2006 président du Pérou s'inscrit dans la même ligne. Voir Carmen Rosa Balbi Scarneo, "Le phénomène Humala", dans Amérique latine, les élections contre la démocratie?, sous la direction d'Olivier Dabène, Paris, 2007, p. 178.

3. El País, 15 septembre 2005.

4. Voir Charles Lancha, Histoire de l'Amérique hispanique de Bolivar à nos jours, p. 268-271.

5. Voir Ch. Lancha, ouvr. cité, p. 469-487.

6. Voir Diego García Monge, "Tareas pendientes de la democratización chilena », revue A-mérika, vol.1, $\mathrm{n}^{\circ}$ 2, 5 décembre 2008, p.1-21. L'auteur analyse les blocages institutionnels à mettre au compte de la droite et la bataille contre l'impunité.

7. El País, 21 décembre 2007.

8. Amartya Sen et Bernardo Kliksberg, Primero la gente, Barcelone, Ediciones Deusto, 2007, p. 195. Près de dix ans plus tôt, Kliksberg stigmatisait déjà l'écart énorme des revenus dans América latina: una Región en riesgo. Pobreza, inequidad e institucionalidad social, disponible sur <http:// www.iigov/pnud:taller/docu/docum13.htm>, octobre 1999, p.18-19. Dans Clarín du 8 octobre 2002, il revenait à la charge : "L'inégalité est la pire caractéristique de l'Amérique latine. » En 2003, un rapport de la Banque mondiale mettait de façon surprenante l'accent sur le caractère de classe de cette particularité : «D'après le rapport, comme au temps du colonialisme, aujourd'hui encore ce sont les élites qui ont donné forme aux institutions et aux politiques publiques en vue de favoriser avant tout leurs propres intérêts. » (<http://wbln0018.worldbank.org/>, 7 octobre 2003.) Cf. la critique véhémente de ce rapport par Mario Wainfeld, <http://www.lpp-uerj.net/ olped/noticias/opiniao_BM.htm>, 11 juillet 2004.

9. A. Sen et B. Kliksberg, ouvr. cité, p. 196.

10. Le bilan dramatique qu'il établit p. 275-276 retient particulièrement l'attention.

11. L'Humanité, 10 juin 2009. 
12. <http://www.rebelion.org>, 17 mai 2006 ; <http://www.taringa.net/posts/noticias>, 2006. Le directeur régional de l'UNICEF pour l'Amérique latine et la Caraïbe, Nils-Arne Kastberg, a souligné l'intérêt qu'attache son organisation à renforcer sa collaboration avec Cuba en vue d'éradiquer la dénutrition chronique en Amérique latine : <http://www.consumer.es/web/es/ solidaridad/>, 6 octobre 2007.

13. La CEPAL distingue deux types de dénutrition: « 8,8 millions d'enfants latino-américains souffrent de dénutrition chronique [...] et 4,2 millions d'enfants souffrent de dénutrition globale. » (Rodrigo Martinez et Andres Fernandez, « Desnutrición infantil en América latina y el Caribe ", Desafíos, Boletín de la infancia y adolescencia sobre el avance de los objetivos de desarrollo del Milenio, $\mathrm{n}^{\circ}$ 2, avril 2006, CEPAL UNICEF, p. 12.) Cf. Angela Cruz Martinez, «Erradicar la desnutrición en México tardará hasta setenta años », La Jornada, 20 janvier 2007. La présidente nationale du DIF estime que $8,9 \%$ de la population infantile au Mexique est soumise à la sous-alimentation, Correo semanal,30 novembre 2007.

14. BBC Mundo.com, 8 juillet 2005.

15. <http://www.esmas.com/>, 22 mars 2006.

16. «La desnutrición infantil en Guatemala es descomunal, denuncia la UNICEF », <http:// www.santafenewmexican.com>, 6 janvier 2008.

17. «Por desnutrición infantil en Bolivia UNICEF pide declaratoria de emergencia nacional», El Diario, La Paz, 14 mai 2008.

18. Elnuevodia.com.co, $1^{\mathrm{er}}$ juin 2009.

19. La democracia en América latina. Hacia una democracia de ciudadanas y ciudadanos, Buenos Aires, Aguilar, Altea, Alfaguara, 2004, PNUD, $1^{\text {re }}$ éd., p. 15.

20. L'Humanité, 12 novembre 2007 ; voir également un article de Michelle Bachelet « Iberoamérica hacia un nuevo pacto social ", El País, 9 novembre 2007. On ne peut s'empêcher de s'interroger sur le caractère rhétorique d'une telle déclaration. En 1999, déjà, Ricardo Lagos, alors candidat à la présidentielle au Chili - il sera élu le 16 janvier 2000 - tenait le même discours: "Les problèmes sociaux qui, supposait-on, devaient battre en retraite, tels que l'aggravation de la pauvreté rurale et urbaine, le maintien et même l'augmentation de la brèche distributive ou la plus grande acuité des problèmes de violence, d'insécurité dans les villes et d'exclusion des jeunes, se posent toujours. L'Amérique latine est la région où la distribution des revenus est la plus inégalitaire au monde. Dans notre région, par conséquent, on doit insister sur la nécessité d'inclure les exclus, d'améliorer la vie de ces derniers sans que ce soit aux dépens des autres. Notre idée est que personne ne doit être perdant dans le processus d'inclusion sociale... » (El País, 19 juillet 1999.) On présente habituellement le Chili comme exemple de réussite du modèle libéral en Amérique latine. Pourtant, la question sociale s'y pose toujours. Selon un certain mode de calcul, en 2006, l'indigence se chiffrait à 3,2 \% et la pauvreté à $13,7 \%$. Selon un autre mode de calcul, conforme aux normes de la CEPAL, plus rigoureux, l'indigence, en 2006, s'élevait à 6,2 \% et la pauvreté à $29 \%$. On comprend mieux les préoccupations des deux chefs d'État chiliens cités cidessus (Andrés Sanfuentes, "Tamaño de la pobreza y la indigencia, contando los pobres » [en ligne], Asuntos públicos, informe $\mathrm{n}^{\circ} 664,25$ septembre 2008, disponible sur <www.ced.cl/ap/wpcontent/uploads/2008/09/664.pdf $>$ ). À la lumière des déclarations de Michelle Bachelet et d'une multitude de dirigeants latino-américains sur la question sociale, on ne peut lire sans quelque surprise sous la plume d'olivier Dabène que «la lutte contre la pauvreté » est "bien engagée grâce aux programmes de transferts monétaires conditionnés qui prolifèrent dans le continent (PROGRESA au Mexique, Bourse Famille au Brésil)» (Amérique latine, les élections contre la démocratie, sous la direction d'Olivier Dabène, Presses de la Fondation nationale, 2007, p. 23). Il est vrai qu'il existe une multitude de plans sociaux, utiles sans doute en général mais la question sociale, comme on vient de le voir, reste entière.

21. Rapport de février 2006.

22. La Nación, 7 novembre 2005. 
23. Amérique latine : les surprises de la démocratie, Paris, La Documentation française, 2007, p. 13. En raison de la crise mondiale qui n'épargne pas la Région, le nombre de pauvres risque de s'accroître de 50 millions.

24. «L'Amérique latine : l'appel d'un continent à maîtriser la globalisation », La Revue socialiste, $\mathrm{n}$ - 27, avril-mai 2007, « La nouvelle donne latino-américaine », p. 39-40.

25. A. Sen et B. Kliksberg, ouvr. cité, p. 238.

26. El País, 27 avril 2005. Luis Esteban G. Manrique rappelle une statistique fondamentale établie par l'OMS : «D'après l'Organisation mondiale de la santé (OMS) le nombre d'homicides annuels par armes à feu en Amérique latine - entre 73000 et 90000 - est trois fois supérieur à la moyenne mondiale [...] Cette dégradation de la sécurité des citoyens a coincidé avec l'affirmation d'un puissant pouvoir parallèle, le crime organisé. » (Real Instituto Elcano de Estudios Internacionales y Estratégicos, 25 juillet 2006.)

27. El País, 28 novembre 1990.

28. La Jornada, 10 juin 2009. Cf. le rapport de la Croix-Rouge internationale à Bogota sur la violence en Colombie en 2008, Página12, 18 avril 2009. Le rapport non seulement dresse un constat des crimes commis contre des civils mais rappelle que, depuis des années, des millions de déplacés vivent dans une situation de détresse totale.

29. José Hilario Lopez Rincon, « 1666 asesinatos aislados », Semanario Virtual, Caja de Herramientas, $n^{\circ}$ 159, 23 mai 2009. En juin, Philip Alston, le rapporteur spécial des Nations unies pour les exécutions extrajudiciaires, s'est rendu en Colombie pour mener une enquête sur les crimes dits «faux positifs » (L'Humanité, 10 juin 2009). Dans une longue déclaration publique, il a fait part de ses premières conclusions (Semanario Virtual, Caja de Herramientas, $n^{\circ} 163,20$ juin 2009).

30. Semanario Virtual, Caja de Herramientas, $n^{\circ} 162,12$ juin 2009. Le compte rendu du rapport fait état d'un jugement de Guy Ryder, secrétaire général de la CSI : « Le fait que certains pays comme la Colombie, le Guatemala ou les Philippines apparaissent tous les ans dans la liste des assassinats, indique que les autorités sont, au mieux, incapables d'assurer une protection adéquate et, parfois, complices d'employeurs sans scrupule dans les assassinats perpétrés. » De fait, chaque année, plus de $60 \%$ des victimes sont colombiennes. Au cours des 23 dernières années, 2709 syndicalistes colombiens ont été abattus.

31. Cf. José Luciano Sanin Vasquez, Director General, Escuela Nacional Sindical, Colombia, «Situación de los derechos de los trabajadores en Colombia. Violencia, impunidad y violación a los derechos laborales y sindicales ", Semanario Virtual, Caja de Herramientas, n 146, 14 février 2009 , p. 3.

32. Cf. Joëlle Stolz, Le Monde, 2 mai 2009.

33. Il existe une bibliographie considérable sur ce thème dramatique. Cf. en particulier Sergio Gonzalez Rodriguez, Huesos en el desierto, Barcelone, Anagrama, 2002, 335 p. ; Charles Lancha, «Jeunes femmes assassinées à Ciudad Juarez (Mexique)», L'Humanité, 15 août 2003 ; Diana Washington Valdez, Cosecha de mujeres. Safari en el desierto mexicano, Editorial Oceano, 2005, 364 p. ; Human Rights Watch, Un caso paradigmático: Ciudad Juárez, mai 2006, 7 p. ; Luz Castro, «Ciudad Juarez : le féminicide continue », Tribune des droits humains, 21 octobre 2008.

34. Margarita Carrera, "Persistencia Feminicidio en Guatemala", Prensa Libre, 17 juin 2005; Amnesty International, Guatemala. Ni protección ni justicia para las mujeres, 14 juillet 2005 ; «Las muertas de Guatemala », Proceso, 18 octobre 2005 ; Amnesty International, Guatemala. Por la vida de las mujeres, ni una muerte más, décembre 2005 ; Mujeres iberoamericanas, Feminicidio en Guatemala, 2 janvier 2006 ; Parlement européen, Los eurodiputados dan visibilidad al problema del feminicidio y buscan estrategia para combatirlo, 21 avril 2006; Adital, Guatemala. Más mujeres asesinadas, 26 avril 2007; Jean-Michel Caroit, « $\mathrm{Au}$ Guatemala, les femmes sont les premières victimes de la violence », Le Monde, 6 septembre 2007.

35. Le Monde, 3-4 avril 2005.

36. El País, 17 avril 2004. Le Monde du 7 août 2004 avance le chiffre de 50000 homicides par an. 
37. El País, $1^{\mathrm{er}}$ octobre 2002.

38. Alternatives économiques, mai 2009, p. 60. Le taux d'homicides oscille entre 1 et 2 pour cent mille.

39. Le Monde, 21 octobre 2000.

40. Histoire du Brésil, 1500-2000, Fayard, 2000, p. 521. Les deux auteurs soulignent l'horreur de la répression à l'égard des enfants de la rue : «Enfants et adolescents misérables sont devenus les cibles privilégiées des sinistres escadrons de la mort qui comptent dans leurs rangs nombre de policiers dévoyés : 1080 exécutions entre janvier 1991 et juillet $1993 »$ (p. 517). C'est ce qu'on appelle dans la région « le nettoyage social » qui a cours dans nombre de pays, en particulier la Colombie et le Guatemala.

41. Le Monde, 19 mars 2008. Dans El País du 2 août 2008, on peut lire à propos de Rio : "Personne n'est capable d'endiguer la violence qui se propage comme un cancer à travers ses rues et ses places. L'aide même de l'armée brésilienne n'a servi à rien. Les milices illégales qui interviennent dans presque 180 favelas et ont commencé à se déployer dans les quartiers riches de la ville ont accru encore davantage l'indice de la violence. » Même situation de violence à Sao Paulo. En 2006, 5276 assassinats dont 413 commis par la Police militaire ; en 2007, 4100 homicides dont 280 perpétrés par la Police militaire ; au cours des neuf premiers mois de 2008, 3690 homicides, dont 296 attribués à la Police militaire. En 2007, la police portugaise a tué une personne et la police anglaise deux. (Cf. La Razón, La Paz, 19 décembre 2008.)

42. El País, 23 août 2000.

43. Amnesty International signale que, en 2005, «les forces de police ont abattu, selon des statistiques officielles, plus de mille civils dans des circonstances qualifiées de "résistance suivie de mort". Ces chiffres n'incluent pas les personnes tuées par des policiers impliqués dans des activités criminelles ou par des membres d'escadrons de la mort » (<http://www.amnesty.fr/>, 1 er septembre 2006).

44. Le Monde, 15 mars 2005.

45. Página12, 29 mars 2005.

46. Le Monde, 27 janvier 2005.

47. El País, 6 mai 2004. Impopularité passagère. Aujourd'hui, tous les sondages lui accordent un indice élevé de popularité. Selon Les Échos du 6-7 février 2009 : «Sa cote de confiance a augmenté de 4 points depuis la fin de l'année dernière pour atteindre $84 \%$ en janvier - le nombre de mécontents a chuté également. » La popularité de Lula a connu bien des vicissitudes (cf. Página12, 25 avril 2009).

48. Les Échos, 6-7 février 2009.

49. Jean-Yves Martin avance la précision suivante : «[...] plusieurs recherches indépendantes ont, ces dernières années, convergé vers une estimation d'un total de Sans-terre d'au moins 3,8 à 4,5 millions de familles au Brésil. Sachant qu'une famille rurale compte en moyenne 4 à 5 personnes, on mesure l'ampleur du problème qui reste donc posé au pays. » (Idelette Muzart Fonseca dos Santos et Denis Rolland [coord.], La terre au Brésil de l'abolition de l'esclavage à la mondialisation, L'Harmattan, 2006, p. 123-124.)

50. Le Monde, 17 mai 2005.

51. La Jornada, 2 mars 2009.

52. Le Monde, 17 mai 2005. L'Humanité du 21 avril 2009 rapporte les statistiques suivantes de l'Instituto Brasileiro de Geografia e Estatistica: «1338711 agriculteurs, soit 31,6\% de la population paysanne possèdent moins de 10 ha, soit $1,8 \%$ des terres cultivées; 32264 agriculteurs, soit $0,8 \%$ de la population paysanne possèdent plus de 2000 ha, soit $31,6 \%$ des terres cultivées. "

53. Le Monde, 24 mai 2005.

54. Página12, $1^{\text {er }}$ février 2009. Sur le MST et la répression qui s'abat sur lui actuellement, cf. Christophe Ventura, "Sans terre mais non sans voix », Le Monde Diplomatique, avril 2009, p. 12. 
55. Thierry Ogier propose un chiffre global de l'allocation : «Les montants versés sont modiques (30 euros par mois en moyenne - ils varient en fonction de la taille de la famille -) [...] de toute évidence la Bolsa Familia ne suffit pas » (Les Échos, 30 avril 2009).

56. Le nouveau Brésil de Lula: dynamique des paradoxes, dirigé par Daniel Van Eeuwen, Éditions de l'Aube, 2006, p. 120-121.

57. El País, 25 décembre 2007.

58. La Revue socialiste, p. 28.

59. Le Brésil au XXI ${ }^{e}$ siècle, Fayard, 2006, p. 313.

60. CEPAL, Panorama social de América latina 2007, p. 57.

61. Human Development Report 2006, Beyon scarity: Power, poverty and the global water crisis, 2006, p. 292.

62. <http://www.elciudadano.cl/2009/02/11/>.

63. < http://www.ine.gob.ve/idh/tablaevolucion.htm>; <http://venciclopedia.com/index.php? title=Índice_de_desarrollo_humano_(ID...>.

64. Julio Labastida Martin del Campo définit clairement la démocratie restreinte propre à ce pays centre-américain : «Au Guatemala, la présence politique de l'armée et la violence des groupes paramilitaires sont un défi à une faible démocratie où la majorité de la population, indigène, est toujours exclue et opprimée. » (Revue Nexos, n³55, juillet 2007.)

65. Les mêmes causes produisant les mêmes effets, les maux dont elles souffrent s'observent de plus en plus dans les démocraties occidentales, elles aussi assujetties à l'économie de marché sans entrave. La différence, elle est de taille, est que les travailleurs latino-américains ne disposent pratiquement pas de couverture sociale et accusent de plein fouet les conséquences de la crise mondiale alors que, en France, comme le soulignait dernièrement l'OFCE - Observatoire français des conjonctures économiques - il existe encore un modèle social qui « dote les ménages français de filets de sécurité plus conséquents ». Au-delà de ces différences, une même crainte d'explosion sociale s'exprime aussi bien en Amérique latine qu'en France. L'ancien Premier ministre Dominique de Villepin vient de déclarer: «Oui, il y a un risque révolutionnaire en France.» Andrés Oppenheimer semble se faire l'écho de Villepin dans un de ses articles : "Une nouvelle étude de la Banque mondiale estime que la crise financière globale engendrera six millions de nouveaux pauvres en Amérique latine cette année. Il s'ensuit des craintes que, outre la récession économique, on assiste à des explosions sociales dans certains pays. » (La Nación, 17 mars 2009.)

\section{RÉSUMÉS}

La problématique de cet article cherche à comprendre comment la démocratie latino-américaine a évolué et son état des lieux aujourd'hui, notamment à la lumière de la relation contradictoire entre permanence des inégalités sociales et processus de consolidation démocratique. Sur le plan institutionnel, les démocraties ibéro-américaines se sont révélées des démocraties «à part entière » alors que la plupart des pays de la région jouissent désormais d'une constitution où les libertés fondamentales sont reconnues. Cependant, au-delà de toutes les entraves existantes à l'affermissement de la démocratie, l'essentiel réside aujourd'hui - selon nous - dans la mise en œuvre pendant un quart de siècle dans toute l'Amérique latine, à l'exception de Cuba, d'un modèle économique néolibéral aux conséquences sociales désastreuses qui a sapé toute possibilité d'affermissement démocratique sans sortir de cet héritage économique. 
The central question this article seeks to understand is how Latin American democracy has evolved and what is its current status today, especially in light of the contradictory relationship between established social inequalities and existing democratic forces. At the institutional level, Ibero-American democracies have proved themselves to be formal democracies, with most countries in the region now having constitutions where fundamental freedoms are legally recognized. However, of all the existing barriers to the establishment of social democracy in Latin American countries, the most important obstacle today, in our view, is the implementation over the past quarter of a century (except in Cuba) of the neoliberal economic model which has had disastrous social consequences and which has until now undermined any possibility of strengthening democracy due to this entrenched economic legacy.

\section{INDEX}

Mots-clés : Amérique latine, démocraties, néolibéralisme, transitions

\section{AUTEUR}

\section{CHARLES LANCHA}

Professeur émérite en Civilisation hispano-américaine à l'université Stendhal-Grenoble 3 